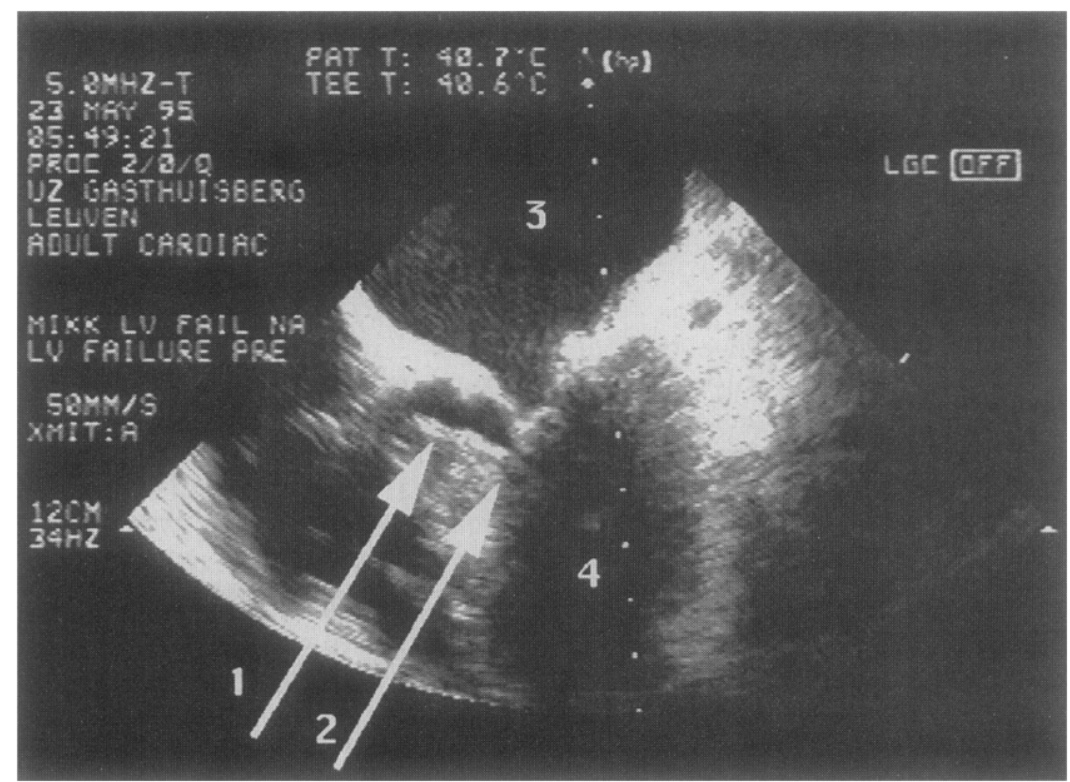

Fig. 1. Two-dimensional transesophageal echocardiography of the left ventricle outflow tract (Arrow 1) after mitral valve repair. Septal anterior motion is obvious, with the tip of the anterior leaflet touching the bulging septum during systole (Arrow 2). Arrow 3, Left atrium; Arrow 4, left ventricle.

3. Schiavone WA, Cosgrove DM, Lever HM, et al. Long-term follow-up of patients with left ventricular outflow tract obstruction after Carpentier ring mitral valvulaplasty. Circulation 1988;78(Suppl 1):I-60-5.

4. Grossi EA, Galloway AC, Parish MA, et al. Experience with twenty-eight cases of systolic anterior motion after mitral valve reconstruction by the Carpentier technique. J Thorac Cardiovasc Surg 1992;103:466-70.

5. Jebara VA, Mihaileanu S, Acar $C$, et al. Left ventricular outflow tract obstruction after mitral valve repair: results of the sliding leaflet technique. Circulation 1993;88(Suppl 2): $30-4$.

\title{
THORACOSCOPIC RELEASE OF TRACHEOPEXY STITCH CAUSING PHRENIC NERVE PARALYSIS IN AN INFANT
}

Rodrigo Oyarzun, MD, Joseph V. Cotroneo, MD, Roberto M. DiDonato, MD, Mary Berry LeBoeuf, MSN, James S. Donahoo, MD, and John R. McCormick, MD, Newark, N.J.

Congenital vascular rings are often complicated by tracheomalacia. Even after release of the tracheal compression, the tracheomalacia may be severe enough to result in postoperative morbidity and mortality. ${ }^{1}$ Therefore aortopexy or tracheopexy, or both, may be required to lessen respiratory insufficiency, especially in the immediate postoperative period. ${ }^{2}$ We observed an unusual

From The Department of Surgery, Division of Pediatric Cardiothoracic Surgery, Children's Hospital of New Jersey, and University of Medicine and Dentistry, New Jersey Medical School, Newark, N.J.

Received for publication Sept. 6, 1995; accepted for publication Oct. 3, 1995. complication-a tracheopexy suture that caused phrenic nerve compression and resulting diaphragmatic paralysis. The phrenic nerve was successfully released by videoassisted thoracoscopic surgery (VATS).

An 8-month-old infant weighing $8.2 \mathrm{~kg}$ was admitted to Children's Hospital of New Jersey for correction of a

\footnotetext{
Address for reprints: J. Rodrigo Oyarzun, MD, University of Medicine and Dentistry of New Jersey-New Jersey Medical School, 185 South Orange Ave., MSB G 595, Newark, NJ 07103.

J Thorac Cardiovasc Surg 1996;112:188-90

Copyright (C) 1996 by Mosby-Year Book, Inc. $0022-5223 / 96 \$ 5.00+0 \mathbf{1 2 / 5 4 / 6 9 6 6 0}$
} 
congenital vascular ring and associated tracheomalacia. The child had had respiratory difficulties shortly after birth. At the age of 10 days laryngomalacia was diagnosed. The patient subsequently had two hospital admissions for treatment of upper respiratory tract infections and bronchiolitis. Eventually, a vascular ring was suspected and a barium swallow confirmed the diagnosis. A nuclear magnetic resonance study revealed a double aortic arch with an atretic anterior segment. Operative correction was then indicated.

After induction of anesthesia, an examination with a rigid bronchoscope demonstrated severe malacia of the anterior tracheal wall at the level of the vascular ring. The left side of the chest was opened by means of a standard posterolateral incision. On exploration, a much larger thymus than normal was noted. The atretic anterior aortic arch and the ligamentum arteriosum were divided. The vessels were then dissected free, which cleared the anterior and lateral tracheal walls from any vascular compression. At the same time, this maneuver provided complete exposure of the trachea. The segment of tracheomalacia was easily identified and suspended to the back of the sternum with a 3-0 nonabsorbable suture. The course of this suture transversed the enlarged thymus, encroaching on the left phrenic nerve, without obvious compression (Fig. 1).

The patient tolerated the procedure well and was extubated immediately after the operation. However, the chest roentgenogram on the second postoperative day showed elevation of the left hemidiaphragm, a condition that worsened over the next 2 days. We attributed this problem to mechanical compression of the left phrenic nerve caused by the suspension stitch. Despite a lack of symptoms, we decided to divide the suture.

To avoid a second thoracotomy, we elected to use a VATS approach. The videoscopic equipment included a Dyonics $4.0 \mathrm{~mm}$ wide-angle 30 -degree arthroscope (Smith \& Nephew Dyonics, Inc., Andover, Mass.). The scope was inserted through the chest tube site with the lungs hypoventilated. A second port (4 mm long) was created at the anterior end of the thoracotomy incision for introduction of instruments. No additional port was necessary, because the scope could also be used to retract the hypoventilated lung. The polypropylene suture was identified and divided with orthopedic endoscopy scissors.

Postoperatively, the left hemidiaphragm resumed function in a matter of hours, as identified by a chest X-ray film and confirmed by means of a fluoroscopic study on the first postoperative day. Although the patient was able to be extubated immediately after the operation, he had persistent problems related to tracheomalacia, which were adequately managed medically. The patient was discharged to his home within a week of the VATS procedure.

Tracheomalacia is often associated with vascular rings and can result in severe respiratory compromise in children. Several surgical techniques have been described to treat severe tracheomalacia. ${ }^{2}$ Depending on the anatomy, either aortopexy or tracheopexy can be performed. Aortopexy was not applicable in this patient, because the only residual aortic arch was posterior to the trachea; thus tracheopexy was chosen. In general, tracheopexy can be performed through either a median sternotomy or a lateral thoracotomy. In cases of tracheomalacia associated with vascular rings, the

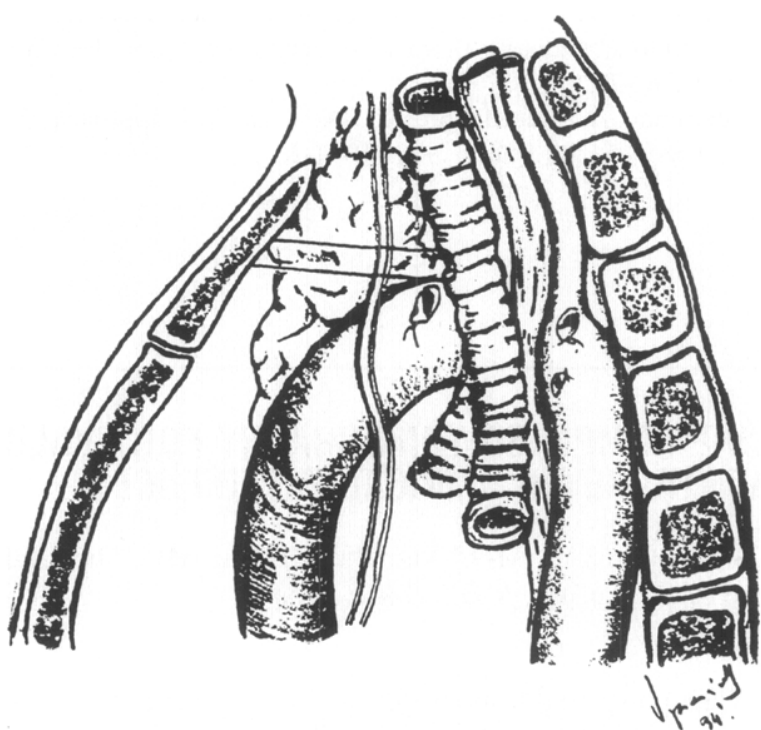

Fig. 1. Schematic left lateral view of the mediastinum after division of the atretic anterior aortic arch (upper two stumps) and of the ligamentum arteriosum (lower stump on the descending aorta). The pericardium, the heart, and the pulmonary artery are not shown. The malacic anterior wall of the trachea is suspended to the back wall of the sternum by a $3-0$ nonabsorable suture. This suture crosses (and partially compresses) the left phrenic nerve, and this compression eventually causes diaphragmatic paralysis.

entire procedure is approached through a left thoracotomy. The trachea is suspended by placing one or more nonabsorbable sutures between the anterior tracheal wall and the sternal periosteum. The procedure is generally well tolerated and operative complications have been unusual. ${ }^{2,3}$

In our patient, hyperplasia of the thymus displaced the phrenic nerve laterally and thus exposed it to compression by the tracheopexy suture. In retrospect, the suspending suture should have been passed through the thymus, medial to the phrenic nerve, which would have avoided its compression and, consequently, diaphragmatic paralysis. This complication often causes life-threatening respiratory insufficiency in infants. Therefore it necessitates early aggressive treatment, which is usually performed through a lateral thoracotomy.

With the recent development of smaller endoscopy instruments, the list of indications for VATS in pediatric cardiothoracic conditions is expanding. ${ }^{5}$ The immediate resolution of the diaphragmatic paralysis and the early recovery of the patient, without the need for a second thoracotomy, is another example of the efficacy of VATS when applied to selected pediatric cases.

REFERENCES

1. Roesler M, de Leval M, Chrispin A, Stark J, Surgical management of vascular ring. Ann Surg 1983;197:139 46. 
2. Filler RM, Tracheomalacia. In: Fallis JC, Filler RM, Lemoine $G$, editors. Pediatric thoracic surgery. New York: Elsevier, 1991:163-71.

3. Vaishnav A, MacKinnon AE. New cervical approach for tracheopexy. Br J Surg 1986;73:441-2.
4. Shoemaker R, Palmer G, Brown JW, King H. Aggressive treatment of acquired phrenic nerve paralysis in infants and children. Ann Thorac Surg 1981;32:251-9.

5. Rogers BM. Pediatric thoracoscopy: Where have we come and what have we learned? Ann Thorac Surg 1993;56:704-7.

\section{CINE COMPUTED TOMOGRAPHY FOR EVALUATION OF TUMORS INVASIVE TO THE THORACIC AORTA: SEVEN CLINICAL EXPERIENCES}

Toshiya Ohtsuka, MD, ${ }^{\mathrm{a}}$ Manabu Minami, MD, ${ }^{\mathrm{b}}$ Jun Nakajima, MD, ${ }^{\mathrm{a}}$ Tadasu Kohno, MD, ${ }^{\mathrm{a}}$ Kuniyoshi Yagyu, MD, ${ }^{\mathrm{a}}$ and Akira Furuse, MD, ${ }^{a}$ Tokyo, Japan

Cine computed tomography (CT) has been reported to be useful for evaluating vessel flow or function of the heart. ${ }^{1-5}$ We have applied it for the preoperative evaluation of intrathoracic tumors. Before operating for an intrathoracic tumor that has been found by conventional $\mathrm{CT}$ to be in contact with the aorta, it is important to judge whether the tumor has invaded the aorta; if this is the case, most surgeons will cancel the operation because of

From the Departments of Cardiothoracic Surgery and Radiology, ${ }^{\mathrm{b}}$ Faculty of Medicine, University of Tokyo, Tokyo, Japan.

Received for publication July 13, 1995; accepted for publication Dec. 13, 1995.

Address for reprints: Toshiya Ohtsuka, MD, Department of Cardiothoracic Surgery, University of Tokyo, 7-3-1 Hongo,

Bunkyo-ku, Tokyo 113, Japan.

J Thorac Cardiovasc Surg 1996;112:190-2

Copyright (C) 1996 by Mosby-Year Book, Inc.

$0022-5223 / 96 \$ 5.00+0 \quad \mathbf{1 2 / 5 4 / 7 1 1 6 9}$

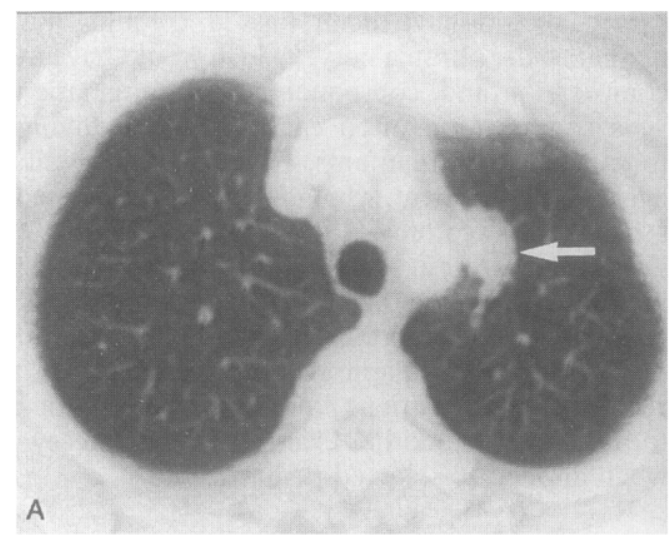

the poor prognosis of a T4 tumor. When a cancer affects the superficial layer of the wall and reveals no sign of invasion by conventional chest $\mathrm{CT}$ or magnetic resonance imaging (MRI), it is virtually impossible to confirm whether malignant adhesion of the tumor exists. To judge preoperatively whether an intrathoracic tumor is invasive to the aorta, we have developed a new method using cine CT. Here we report seven clinical experiences with this new method.

Starting in June 1992, after institutional review board approval and written informed patient consent had been obtained, cine CT was applied for seven consecutive patients with left intrathoracic tumors in contact with the aortic wall. All of the seven tumors, six pulmonary and one thoracoparietal, had been identified previously by conventional chest CT and MRI (Fig. 1). The portions in contact with the seven tumors ranged from the distal arch to the descending aorta. Three pulmonary tumors and a thoracoparietal tumor were adjacent to the distal arch and three lung tumors touched the descending aorta on each side of the mediastinum. Furthermore, one of the lung tumors had invaded the distal arch, which showed obvious deformity and an irregular wall boundary, whereas no

Fig. 1. Standard chest CT scan (A) of tumor 7 (arrow) showing that the tumor was in contact with the aortic wall. B, CT scan of tumor 1. 